DOI: https://doi.org/10.46296/ig.v1i1.0001

\title{
OBTENCIÓN DE ALCOHOL ETÍLICO A PARTIR DE DOS VARIEDADES DE CABUYA: ALTERNATIVA PARA SU INDUSTRIALIZACIÓN
}

\section{OBTAINING OF ETHYL ALCOHOL FROM TWO VARIETIES OF CABUYA: ALTERNATIVE FOR ITS INDUSTRIALIZATION}

\author{
Loor-Ponce Dimas ${ }^{1 *}$ \\ ${ }^{1}$ Universidad Laica Eloy Alfaro de Manabí, ULEAM. Manta, Ecuador.
}

*Correo: dimas.loor@gmail.com

\begin{abstract}
Resumen
Este proyecto de investigación estudia la utilización de plantas nativas de Furcraea cabuya var. integra y Furcraea cabuya var. cabuya del litoral ecuatoriano, cosechadas en los poblados de: Ayampe, Montecristi, Tosagua, Portoviejo, con el objetivo principal de obtener alcohol etílico mediante la fermentación, destilación y filtración y de esta forma conseguir una bebida alcohólica apta para el consumo humano. En el acondicionamiento y desarrollo experimental se consideran los diferentes parámetros como los diferentes tiempos y temperaturas de cocción, las pruebas con diferentes tipos de inóculos y concentraciones de levaduras, además de diferentes suplementaciones nutritivas (con especial atención a la fuente de nitrógeno). Estableciendo como temperatura óptima para la hidrólisis de la inulina $117^{\circ} \mathrm{C}$ durante 4 horas y el proceso fermentativo óptimo utilizando levadura seca activa comercial al $1 \% \mathrm{p} / \mathrm{v}$ con respecto al mosto y una suplementación con sulfato de amonio de $0,15 \mathrm{~g} / 100 \mathrm{~mL}$ de mosto. El mosto de cabuya rectificado genera una bebida con exquisito sabor a Tequila, la cual luego de ser evaluada sensorialmente demostró que el licor producido se encuentra dentro de los parámetros de aceptación de los consumidores.
\end{abstract}

Palabras clave: etanol, cabuya, destilación, fermentación, mosto, tequila.

\begin{abstract}
This research project studies the use of native plants of Furcraea cabuya var. integra and Furcraea cabuya var. cabuya from the Ecuadorian coast harvested in the towns of: Ayampe, Montecristi, Tosagua, Portoviejo, with the main objective of obtaining ethyl alcohol through fermentation, distillation and filtration in order to achieve an alcoholic beverage suitable for human consumption. In the conditioning and experimental development, the different parameters are considered, such as the different cooking times and temperatures, the tests with different types of inocula and yeast concentrations, as well as different nutritional supplements (with special attention to the nitrogen source). Establishing optimum temperature for the hydrolysis of inulin $117^{\circ} \mathrm{C}$ for 4 hours and the optimal fermentation process using commercial active dry yeast at $1 \% \mathrm{w} / \mathrm{v}$ with respect to the must and a supplementation with ammonium sulfate of $0.15 \mathrm{~g} / 100 \mathrm{~mL}$ of must. The rectified cabuya must gives us a drink with an exquisite Tequila flavor, which after being sensorially evaluated showed that our liquor is within the parameters of consumer acceptance.
\end{abstract}

Keywords: ethanol, cabuya, distillation, fermentation, must, tequila.

Información del manuscrito:

Fecha de recepción: 19 de octubre de 2017

Fecha de aceptación: 06 de diciembre de 2017

Fecha de publicación: 10 de enero de 2018 


\section{Introducción}

Las dos variedades de Furcraea cabuya más comunes en Ecuador son la Furcraea cabuya var. cabuya y la Furcraea cabuya var. integra. La primera variedad ocupa la mayor proporción de los cultivos, porque las fibras de la hoja son muy adecuadas para la fabricación de cuerdas, cordeles, tapetes, sacos, hamacas, alforjas y artesanías. La Furcraea cabuya var. integra comúnmente se encuentra sembrada, como linderos entre fincas 0 cercas vivas de potreros, y no ha sido explotada para la extracción de fibras.

Según Meza (2011) la cabuya o agave tiene una gran variedad de utilidades, como lo es la piña de la agave negra que se utiliza para la elaboración miel de agave y el tequila debido a la abundante cantidad de azúcares que contienen, los cuales le permiten fermentar rápidamente. Mientras que la cabuya blanca o sisal se utiliza para la elaboración de fibras para cordelería, cinturones, bolsos, entre otros accesorios (Rubio \& Soto, 2015).

Según Téllez et al., (2012) el proceso de fermentación es muy importante debido a que involucra biotransformación de azúcares en diversos alcoholes y compuestos aromáticos, que permiten otorgar a cada bebida su identidad distintiva en olor y sabor; es por ello que las levaduras desempeñan un papel fundamental. No obstante, los tipos de cepas, composición, concentración del medio y las condiciones de fermentación, inciden en la biosíntesis de los alcoholes.

En la industria es común la adición de una fuente de nitrógeno en mostos debido a que se desconoce la composición de sus nutrientes y así favorecer el metabolismo de la levadura, de tal manera se evita que este desbalance afecte la composición y el rendimiento del producto final, es decir, una baja producción de alcohol, con mayor cantidad de Agave y alteración de la calidad de la producción de compuestos sensoriales, generando compuestos no deseados (Arellano et al., 2008).

Un factor importante a contemplar y que interviene en las condiciones del medio de cultivo es la temperatura, ya que afecta en el metabolismo de la levadura, lo cual, provocaría la producción de metabolitos 
secundarios. Según Téllez et al., (2012) en el caso de los vinos y/o producción de cerveza, las temperaturas por arriba de los $20^{\circ} \mathrm{C}$ favorecen la producción de alcoholes superiores, esteres y ácidos grasos, mientras que temperaturas que oscilan entre los $5^{\circ} \mathrm{C}$ y $15^{\circ} \mathrm{C}$ favorecen particularmente la producción de acetaldehído y diacetilo.

Por lo mencionado anteriormente, en este trabajo se da a conocer la metodología para la obtención de alcohol etílico a partir de dos variedades de cabuya, como alternativa para su industrialización.

\section{Metodología}

\subsection{Diseño metodológico}

\subsubsection{Variedades seleccionadas de sustrato}

La variedad íntegra es plantada como cerca viva o como ornamental y ambas variedades contienen azúcares concentrados en su tallo, conocido como piña. Sin embargo, se seleccionaron dos variedades de cabuya, que crecen espontáneamente en todo el litoral ecuatoriano, estas variedades son; Furcraea cabuya var. cabuya y Furcraea cabuya var. integra.

\subsubsection{Selección y cosecha}

Las plantas de la variedad Furcraea cabuya var. integra se obtuvieron en el poblado de Ayampe, Ecuador. Se seleccionaron cuatro plantas al azar en etapas fenológicas distintas, con una graduación máxima de 17,0 -Brix. Cabe destacar que al no existir plantaciones dedicadas a este cultivo, la localización de plantas de esta variedad en un estado de madurez fisiológica ideal para el estudio, fue difícil. En ambas variedades se cosecharon piñas de forma ovoide hasta un peso de 100 lb por unidad $(45,359 \mathrm{Kg})$.

\subsubsection{Cocción de la cabuya}

Se realizaron pruebas con ambas variedades de la cabuya, utilizando muestras de $7 \mathrm{~kg}$ de peso a diferentes temperaturas de autolavado $\left(100,110,117\right.$ y $\left.124^{\circ} \mathrm{C}\right)$ durante 4 horas en todos los casos, para así conocer el tiempo y la temperatura óptima de cocción de la cabuya y de esta forma realizar la hidrólisis de los azúcares correspondientes (inulina).

\subsubsection{Preparación del mosto}

Se homogeneizó el mosto y se introdujo en los recipientes para cada uno de los diseños 
experimentales. Con la finalidad de evitar contaminaciones de microorganismos ambientales que interfieran en los procesos fermentativos durante los diferentes ensayos, el mosto fue nuevamente esterilizado a $121^{\circ} \mathrm{C}$ durante $15 \mathrm{~min}$.

\subsection{Análisis y descripción experimental}

La presente investigación fue desarrollada con base a tres diseños experimentales, cada uno de ellos con una finalidad específica:

\subsubsection{Primer diseño experimental:} selección del inóculo inicial (tipo de levadura)

Se emplearon dos variedades de cabuya (Furcraea cabuya var. integra y Furcraea cabuya var. cabuya), con dos tipos de levadura (Saccharomyces cerevisiae) marca comercial (Levapan); Levadura Seca Activa (LSA) y Levadura Fresca (LF).

Para llevar a cabo las pruebas fermentativas, previamente se realizó la preparación del mosto. Posteriormente, el mosto estéril fue inoculado por duplicado con las dos fuentes de levaduras (LSA y LF) en dos formulaciones diferentes: precultivada y sin pre-cultivar.

\subsubsection{Preparación de levaduras} pre-cultivadas

Ambos tipos de levaduras (LSA y LF) se cultivaron con $100 \mathrm{~mL}$ de medio de cultivo Papa Sacarosa (PS) en Erlenmeyers de $250 \mathrm{~mL}$ de capacidad durante 3 días a $25^{\circ} \mathrm{C}$ y sin agitación. De esta forma se obtuvo una suspensión celular final a una concentración de 1x108 células $/ \mathrm{mL}$. Esta suspensión celular fue inoculada por duplicado en recipientes con $100 \mathrm{~mL}$ de mosto de ambas variedades de cabuya previamente hidrolizados, a dos concentraciones diferentes: 2 y 20 $\mathrm{mL}$.

\subsubsection{Preparación de levaduras sin pre-cultivar}

Para este caso se inocularon recipientes con $100 \mathrm{~mL}$ de mosto de ambas variedades de cabuya hidrolizada, con LSA y LF sin modificar (con respecto a su presentación comercial), a una concentración del 1\% p/v.

\subsubsection{Segundo diseño} experimental: selección de la concentración óptima de levadura

Se emplearon los mostos hidrolizados de las dos variedades de cabuya esterilizados. Se 
inocularon tres dosis diferentes de LSA $(0,4 \%, 0,7 \%$ y $1 \% \mathrm{p} / \mathrm{v}$ de $S$. cerevisiae) de forma directa (es decir sin pre-cultivar). Asimismo, se realizó un tratamiento testigo con $0 \%$ de levadura. Para llevar a cabo las pruebas fermentativas, se preparó el mosto y posteriormente, el mosto estéril fue inoculado por triplicado.

\subsubsection{Tercer diseño experimental:} adición de sulfato de amonio en la fermentación

Se emplearon los mostos de las dos variedades de Cabuya y se llevaron a concentraciones de 11 y 22 Brix iniciales por medio de un balance de materia, por ende, se calcularon cuántos gramos de azúcar fermentable (sacarosa) se debían agregar al mosto para llegar a una concentración de 22 Brix, por otro lado, para obtener mostos a 11ํㅡㄹ (menor concentración), se realizaron diluciones con agua destilada estéril. Antes de realizar las pruebas fermentativas se realizó la inoculación de las levaduras y el sulfato de amonio, se adhirió $100 \mathrm{~mL}$ de mosto hidrolizado en concentraciones de 11 y 22 Brix, y se procedió a esterilizar.

Posteriormente, el mosto de ambas variedades de cabuya hidrolizada, se inoculó con LSA (sin pre-cultivar), a una concentración del 1\% p/v. Luego se agregó Sulfato de Amonio (fuente de nitrógeno para las levaduras), a razón de 0,1 g/ $100 \mathrm{~mL}$ al sustrato.

Paralelamente, se realizó un medio de cultivo artificial para evaluar la dinámica poblacional de las levaduras y su capacidad fermentativa. Para ello se preparó una solución concentrada del medio de cultivo Czapek con los siguientes componentes: nitrato de sodio $30 \mathrm{~g}$, cloruro de potasio $5 \mathrm{~g}$, sulfato de magnesio $5 \mathrm{~g}$, sulfato ferroso $0,1 \mathrm{~g}$, agua destilada $100 \mathrm{~mL}$. El medio de cultivo concentrado se esterilizó a $121^{\circ} \mathrm{C}$ durante 15 minutos y se conserva en oscuridad.

Antes de realizar la inoculación de la solución concentrada de Czapek y de las levaduras, se prepararon recipientes de vidrio con tapa metálica de $200 \mathrm{~mL}$ de capacidad y se adhirió $100 \mathrm{~mL}$ de agua destilada a cada uno. Posteriormente, estos recipientes se dividieron en dos grupos. A uno de ellos se le agregó sacarosa hasta lograr un valor de refractometría de $11^{\circ}$ Brix, y al otro grupo se le agregó sacarosa hasta obtener un valor una concentración de 22 oBrix. A continuación, los 
recipientes fueron autoclavados a $121^{\circ} \mathrm{C}$ durante $15 \mathrm{~min}, \mathrm{y}$ por último se adicionaron $10 \mathrm{~mL}$ de la solución concentrada del medio Czapek y LSA sin pre-cultivar ( $1 \% \mathrm{p} / \mathrm{v})$.

\subsection{Control de procesos operacionales}

\subsubsection{Control del proceso de destilación}

Una vez evaluadas las diferentes variables que afectan la fermentación (de acuerdo a lo expuesto en los experimentos 1, 2 y 3), se continuó con el proceso de elaboración (destilación y rectificación) a mayor volumen. Para ello de acuerdo a los resultados obtenidos durante las pruebas fermentativas, se realizó una fermentación con 39,00 litros de mosto de Furcraea cabuya var. integra con una concentración de azúcares iniciales de 23,5ํㅗix (suplementado con sacarosa) y adición de sulfato de amonio $(0,15$ $\mathrm{g} / 100 \mathrm{~mL})$. Una vez finalizada la fermentación se procedió a la destilación y rectificación correspondiente.

En la presente investigación, los destiladores utilizados no contaban con sistema automático de control de tiempo y temperatura, por lo tanto, se trabajó de manera cuidadosa con la rectificación, llevando un control de temperatura manual.

La eliminación de los "picos" (metanol, isopropanol, acetato de etilo y algunos ésteres) se realizó regulando la temperatura hasta los $78,3 \stackrel{\circ}{\mathrm{C}}$ manteniendo destapado el equipo. Los principalmente alcoholes superiores y furfurales se eliminaron a temperaturas superiores a los $96^{\circ} \mathrm{C}$. El "cuerpo" de la destilación se obtuvo entre los 78,4 y $95^{\circ} \mathrm{C}$.

\subsubsection{Análisis físico-químicos}

Para la obtención del alcohol etílico de cabuya se utilizó la norma nacional: Alcohol Etílico Rectificado INEN 375 (tabla 1).

\section{Tabla 1.}

Requisitos del alcohol etílico rectificado según norma INEN 375

\begin{tabular}{|l|c|c|c|c|}
\hline \multicolumn{1}{|c|}{ Requisitos } & Unidad & Mín. & Máx. & $\begin{array}{c}\text { Método de } \\
\text { ensayo }\end{array}$ \\
\hline Grado alcohólico a $15^{\circ} \mathrm{C}$. & ${ }^{\circ} \mathrm{GL}$ & 95 & - & INEN 340 \\
\hline Acidez volátil, como ácido acético. & $*$ & - & 10 & INEN 341 \\
\hline Esteres, como acetato de etilo. & $*$ & - & 5 & INEN 342 \\
\hline Aldehídos, como etanal & $*$ & - & 2 & INEN 343 \\
\hline Furfural. & $*$ & - & 0 & INEN 344 \\
\hline Alcoholes superiores. & $*$ & - & 5 & INEN 345 \\
\hline Metanol. & $*$ & - & 8 & INEN 347 \\
\hline Tiempo de perm anganato & min & 20 & - & INEN 1546 \\
\hline Congéneres & $*$ & - & 18 & \\
\hline$*$ mg/100 cm3 de alcohol anhidro. & & & \\
\hline
\end{tabular}

Esta norma define al alcohol etílico rectificado. Es el producto obtenido mediante destilación y rectificación 
de mostos que han sufrido fermentación alcohólica, como también el producto de la rectificación de aguardientes naturales y que puede utilizarse en la elaboración de bebidas alcohólicas.

\subsubsection{Análisis microbiológicos}

Para garantizar la inocuidad del producto, los parámetros establecidos para productos de consumo humano se detallan en la tabla 2.

Tabla 2.

Características microbiológicas para el licor de cabuya

\begin{tabular}{|l|c|c|c|}
\hline \multicolumn{1}{|c|}{ Parámetros } & m & M & C \\
\hline Recuento de microorganismos mesofilicos & 100 & 300 & 1 \\
\hline NMP coliformes totales/cc & $<3$ & - & 0 \\
\hline NMP coliformes fecales/cc & $<3$ & - & 0 \\
\hline Recuento de Hongos y levaduras/cc & $>10$ & 100 & 1 \\
\hline $\begin{array}{l}\text { m = Índice máximo permisible para identificar nivel de buena } \\
\text { calidad; M Índice máximo permisible para identificar nivel } \\
\text { de aceptable calidad; c = Número máximo de muestras } \\
\text { permisibles con resultado entre m y M; NMP = Número más } \\
\text { probable. }\end{array}$
\end{tabular}

Para estos análisis se procedió a utilizar placas de Petrifilm, cada una con su especificación técnica, se realizaron pruebas por triplicado a cada una de las muestras del licor de cabuya obtenido a partir de Furcraea cabuya var. integra. Se utilizó en este estudio el licor rectificado de cabuya en concentración inicial de $40 \%$ vol/alcohol, cada placa de Petrifilm fue incubada cumpliendo con las normas establecidas de bromatología y el uso del laboratorio para garantizar el análisis en las placas de:

- Placa $3 M^{\mathrm{TM}}$ Petrifilm ${ }^{\mathrm{TM}}$ para recuento de aerobios mesofílicos.

- Placa $3 \mathrm{M}^{\mathrm{TM}}$ Petrifilm ${ }^{\mathrm{TM}}$ para recuento de Coliformes totales.

- Placa $3 \mathrm{M}^{\mathrm{TM}}$ Petrifilm ${ }^{\mathrm{TM}}$ para recuento de Hongos y Levaduras.

\section{Resultados y discusión}

\subsection{Selección del inóculo inicial:} tipo de levadura

\subsubsection{Cuantificación y} caracterización micromorfológica de las levaduras en los mostos durante el proceso fermentativo

El mosto fermentado de ambas variedades de cabuya, fue analizado por medio de un microscopio óptico y sometido a conteo en microscopio luego de 166 horas de fermentación; determinando de esta forma, la concentración de células con características morfológicas similares a Saccharomyces cerevisiae y el porcentaje de células gemando. Además, permitió determinar el grado de contaminación de cada muestra y el estado fisiológico de las células. 


\subsubsection{Ensayos con levaduras pre-cultivadas}

La interacción entre estos factores al utilizar levadura pre-cultivada, arrojó los resultados que se presentan en las figuras 1 y 2 .

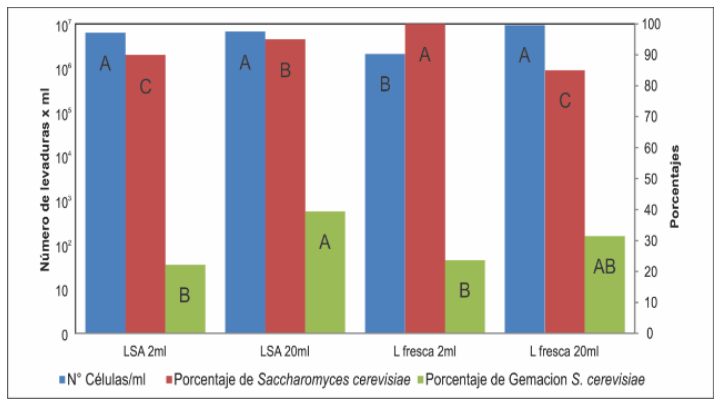

Figura 1. Cuantificación y caracterización micromorfológica de las levaduras en mostos de Furcraea cabuya var. integra con diferente tipo y dosis de inóculo. *Letras diferentes para cada variable dependiente $\left(N^{\circ}\right.$ de células, \% de $S$. cerevisiae $0 \%$ de gemación de $S$. cerevisiae) indican diferencia significativa a $P \leq 0,05$ entre cada tipo de inóculo.

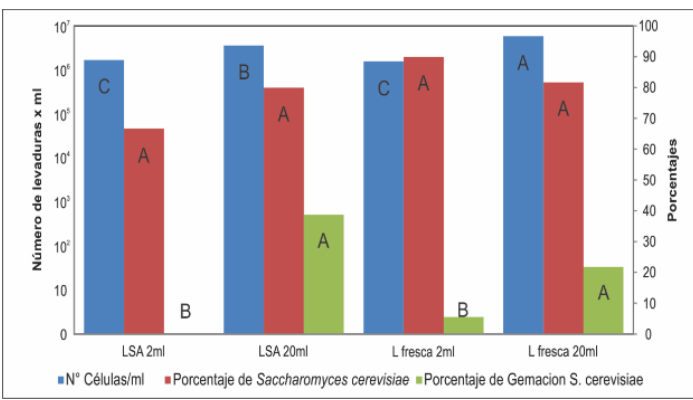

Figura 2. Cuantificación y caracterización micromorfológica de las levaduras en mostos de Furcraea cabuya var. cabuya. con diferente tipo y dosis de inóculo. *Letras diferentes para cada variable dependiente $\left(N^{\circ}\right.$ de células, \% de $S$. cerevisiae $0 \%$ de gemación de $S$. cerevisiae) indican diferencia significativa a $P \leq 0,05$ entre cada tipo de inóculo.
De acuerdo a los resultados presentados en las figuras 1 y 2 , se puede observar una alta concentración de células $/ \mathrm{mL}$ y un alto porcentaje de célula $S$. cerevisiae $(>80 \%$ en la variedad integra; $>65 \%$ en la variedad cabuya). Sin embargo, el porcentaje de células gemando no superó el $40 \%$ en ninguno de los tratamientos. Este resultado puede deberse a la alta concentración celular y a la alta competencia por nutrientes, lo que induciría a las células a disminuir su tasa reproductiva.

Por otro lado, cabe destacar que no se observaron diferencias significativas a favor del tratamiento inoculado con $20 \mathrm{~mL}$ de levadura fresca en la variedad integra, tal y como se manifiesta durante la evaluación de los grados Brix (Fig. 3). Por tal motivo, el mayor consumo de azúcares manifestado en este tratamiento (L Fresca $-20 \mathrm{~mL}$ ) no se debió a un mayor número de células $/ \mathrm{mL}$, y probablemente el mismo fue consecuencia de otro factor fisiológico o metabólico celular no evaluado en el presente trabajo. 


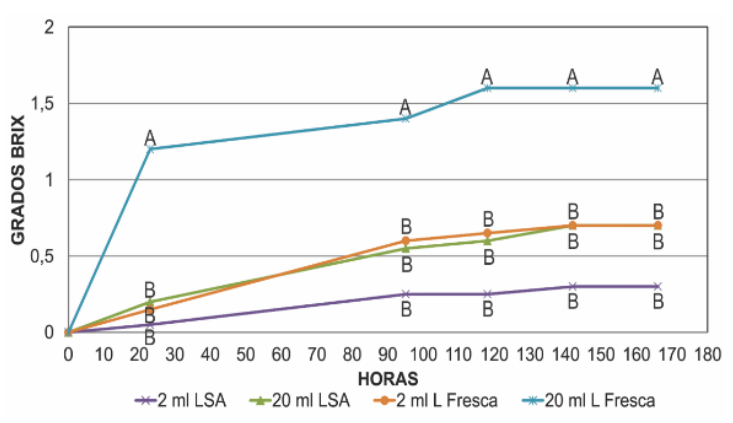

Figura 3. Evolución del consumo de azúcares (determinado en ${ }^{\circ}$ Brix) durante la fermentación de Furcraea cabuya var. integra, con dos tipos de levadura precultivada (Levadura Seca Activa y Levadura Fresca) y dos concentraciones $(2$ y $20 \mathrm{~mL} / 100 \mathrm{~mL}$ de mosto).

*Letras diferentes para cada tiempo de muestreo (23, 95, 118, 142 y 166 horas) indican diferencia significativa a $P \leq 0,05$.

\subsubsection{Ensayos con levaduras pre-cultivadas}

Para este análisis se tomaron en cuenta ambas variedades de cabuya: Furcraea cabuya var. integra y Furcraea cabuya var. cabuya. Los mostos de ambas variedades fueron inoculados con un $1 \% \mathrm{p} / \mathrm{v}$ de levadura en ambas formulaciones (LSA y LF). Al igual que lo expresado en el punto anterior, se realizó el conteo en microscopio para los factores: células por mililitro, porcentaje de Saccharomyces cerevisiae y porcentaje de células gemando. De esta manera se pudo determinar cuál es el tipo de levadura óptimo para la obtención de alcohol etílico, la interacción entre estos factores es presentada en la figura 4.

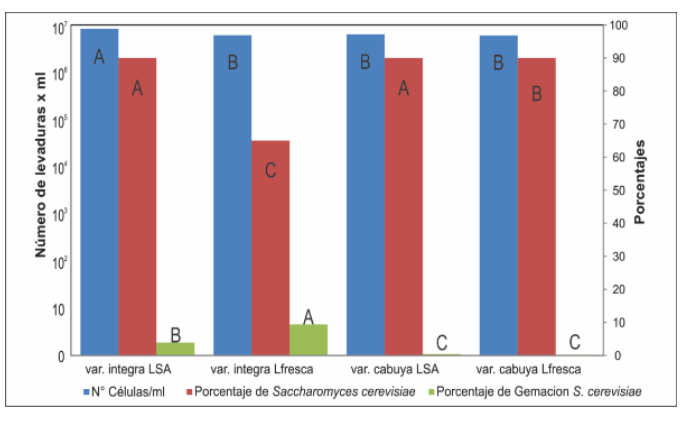

Figura 4. Cuantificación y caracterización micromorfológica de las levaduras en mostos de Furcraea cabuya var. cabuya y Furcraea cabuya var. integra con diferente tipo de inóculo. *Letras diferentes para cada variable dependiente $\left(N^{\circ}\right.$ de células, \% de $S$. cerevisiae $0 \%$ de gemación de $S$. cerevisiae) indican diferencia significativa a $P \leq 0,05$ entre cada tipo de inóculo y variedad de cabuya.

En la figura anterior se observa que los resultados obtenidos durante el registro de las variables micromorfológicas no se corresponden en su totalidad con los resultados observados durante la determinación del consumo de azúcares (Fig. 5).

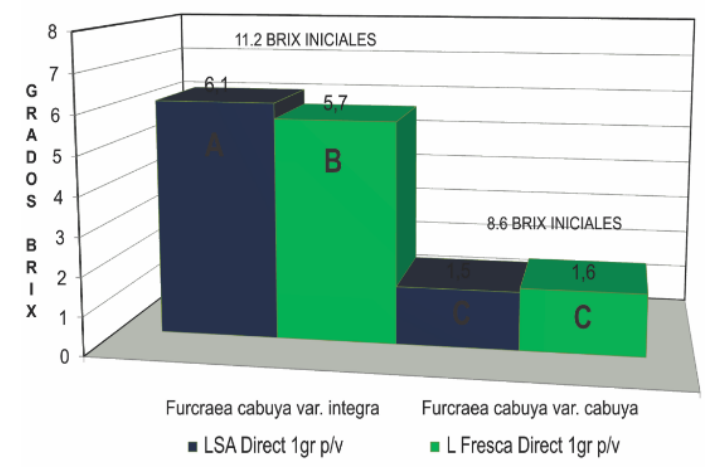

Figura 5. Consumo de azúcares ( ${ }^{\circ}$ Brix) finales en ambas variedades de cabuya $y$ dos tipos de levadura al $1 \% \mathrm{p} / \mathrm{v}$. 
Durante la determinación de los grados Brix (Fig. 5), se observó un consumo de azúcar mayor (diferencia altamente significativa) en los mostos de la variedad integra fermentados con LSA.

En la figura 4, se puede observar que el mayor registro de células $/ \mathrm{mL}$ también fue detectado en el mismo tratamiento, sin embargo, el resto de las variables medidas no arroja el mismo resultado. Esta inconsistencia en los datos, podría deberse a que los factores que determinan el mayor consumo de azúcar se deben a variables no evaluadas en este trabajo (p. ej. características metabólicas).

\subsection{Selección de la concentración} óptima de levadura

En el diseño experimental, se demostró que la levadura seca activa (LSA) presentó el mejor comportamiento fermentativo, de acuerdo a la evolución del consumo de azúcares registrado. Por tal motivo, para determinar la concentración óptima se ensayaron diferentes concentraciones. Las dosis utilizadas fueron: 0,4\%, 0,7\% y $1 \% \mathrm{p} / \mathrm{v}$ de Saccharomyces cerevisiae sin pre-cultivar más un tratamiento testigo con $0 \%$ levadura para evaluar el efecto de la levadura. Todos los tratamientos se realizaron por triplicado.

Los resultados del análisis son presentados por separado para cada una de las variedades: Furcraea cabuya var. integra y Furcraea cabuya var. cabuya. Para posteriormente, realizar la interpretación y discusión del diseño experimental por separado.

\subsubsection{Furcraea cabuya var. integra}

Cada unidad experimental fue monitoreada llevando el control del consumo de grados Brix en relación al tiempo transcurrido en horas. El análisis de varianza se realizó en cada punto de muestreo.

\subsubsection{Furcraea cabuya var. cabuya}

El resultado experimental para ambas variedades de cabuya (Furcraea cabuya var. integra y Furcraea cabuya var. cabuya) demostraron que el mejor tratamiento fue el inóculo con $1 \% \mathrm{p} / \mathrm{v}$ de LSA, donde el consumo de los azúcares fue mayor.

\subsubsection{Consumo total de grados} oBrix: diferentes dosis de levadura

En la Figura 6, se puede observar la diferencia de consumos de azúcar 
entre ambas variedades de cabuya, Furcraea cabuya var. integra y Furcraea cabuya var. cabuya. EI mayor consumo de grados Brix fue para la variedad integra. La variedad cabuya no fermentó lo suficiente, para poder obtener una cantidad considerable de alcohol y ser utilizada para los ensayos de destilación.

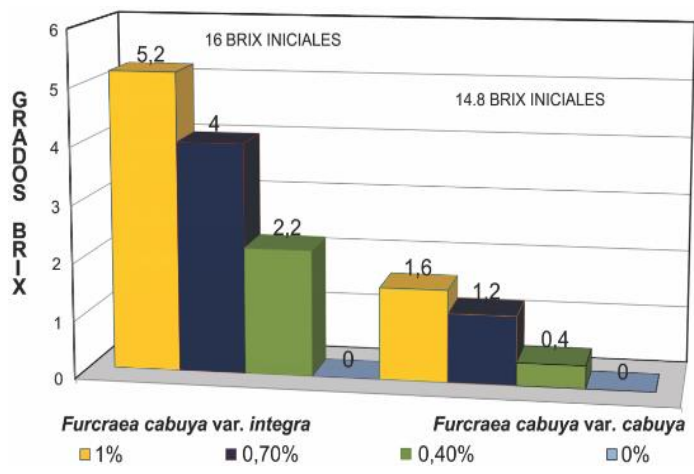

Figura 6. Consumo de azúcares (ํㅗix) final entre las variedades de cabuya, con cuatro dosis diferentes.

\subsection{Adición de sulfato de amonio en la fermentación}

\subsubsection{Análisis de la interacción entre Furcraea cabuya var. integra y Furcraea cabuya var. cabuya con adición de nitrógeno.}

Con este diseño experimental se determinó el efecto comparativo entre variedades al suplemento con nitrógeno (Fig. 7).

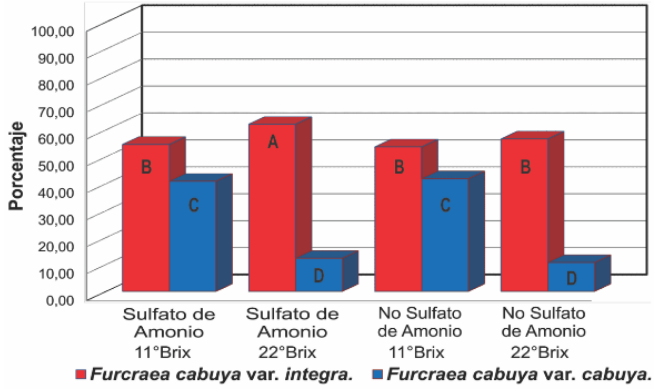

Figura 7. Análisis comparativo entre ambas variedades para el porcentaje de azúcar consumida con respecto al inicio de la fermentación, en mostos con y sin suplementación nitrogenada $(0,1$ g/100 $\mathrm{mL}$ sulfato de amonio). Las concentraciones de azúcares iniciales fueron 11 y $22^{\circ}$ Brix. Las fermentaciones se inocularon con un $1 \%$ de LSA. Letras diferentes indican diferencia significativa a $P \leq 0,05$.

En este análisis se observó nuevamente la mayor capacidad fermentativa de la variedad Furcraea cabuya var. integra sobre la variedad Furcraea cabuya var. cabuya. Asimismo, también se confirma la necesidad de realizar una suplementación nitrogenada en la variedad Furcraea cabuya var. integra cuando la fermentación se inicia con $22^{\circ}$ Brix. Este resultado, demuestra la necesidad de realizar la fermentación con un balance adecuado de la relación $\mathrm{C} / \mathrm{N}$.

\subsection{Análisis físico-químicos}

La muestra de licor de cabuya rectificado, que se obtuvo a partir de la fermentación del mosto de Furcraea cabuya var. integra, con 
inóculo al 1\% p/v de LSA y con el uso de nutrientes a razón de $1,5 \mathrm{~g} / \mathrm{L}$ de sulfato de Amonio presentó las siguientes características.

Tabla 3. Evaluación físico-química del licor de cabuya

\begin{tabular}{|l|c|}
\hline Parámetros & Resultados \\
\hline${ }^{\circ}$ Brix & 0 \\
\hline $\mathrm{pH}$ & 4 \\
\hline $\begin{array}{l}\text { Contenido alcohólico } 20^{\circ} \mathrm{C} \\
(\% \text { Vol.) }\end{array}$ & 40 \\
\hline $\begin{array}{l}\text { Acidez volátil* } \\
\text { (mg/100 cc) }\end{array}$ & 0.05 \\
\hline $\begin{array}{l}\text { Extracto Seco } \\
\text { (g/l) }\end{array}$ & 0.09 \\
\hline $\begin{array}{l}\text { Alcoholes Superiores* } \\
(\mathrm{mg} / 100 \mathrm{~mL})\end{array}$ & 20 \\
\hline $\begin{array}{l}\text { Metanol } \\
(\mathrm{mg} / 100 \mathrm{~mL})\end{array}$ & $<2,5$ \\
\hline $\begin{array}{l}\text { Aldehídos (acetaldehído) } \\
(\mathrm{mg} / 100 \mathrm{~mL})\end{array}$ & 10 \\
\hline $\begin{array}{l}* \text { Alcoholes de mayor peso molecular que } \\
\text { el etanol. }\end{array}$ & \\
\hline
\end{tabular}

El licor obtenido a partir de la destilación del mosto fermentado de Furcraea cabuya var. integra, se encuentra dentro de los rangos permitidos para un licor blanco conocido como "Tequila", ya que fue elaborado con un $49 \%$ de azucarares fermentables externos y un $51 \%$ de azúcares de la planta y sin maduración ni añejamiento, de acuerdo a lo que exige la Norma Mexicana de Licores de Agave.

En Ecuador no existe una normalización para este tipo de alcohol, ya que no hay plantas dedicadas a la explotación de este recurso, por lo tanto, a través de una comunicación personal con expertos del Instituto Ecuatoriano de Normalización (INEN) se recomendó aplicar la Norma INEN 375.

\subsection{Análisis microbiológicos}

Después de transcurrido el tiempo de incubación para cada placa de Petrifilm, se procedió al conteo de las muestras, tres placas para Coliformes totales Método oficial 991.14; tres placas para recuento de Hongos y Levaduras AOAC Método oficial 997.02 y tres placas para aerobios mesofílicos AOAC Método oficial 966.23.

Se demuestra que el producto destilado obtenido no presentó contaminación microbiana, confirmando la hipótesis de que en medios alcohólicos no existe contaminación microbiana. En la tabla 4 se exponen estos resultados.

Tabla 4. Caracterización microbiológica del producto destilado

\begin{tabular}{|c|c|c|c|c|}
\hline Licor & Prueba solicitada & Unidad & Resultados & Método de ensayo \\
\hline \multirow{3}{*}{ Muestra 1} & $\begin{array}{c}\text { Determinación de } \\
\text { Coliformes }\end{array}$ & $\mathrm{UFC} / \mathrm{mL}$ & $<1^{*}$ & $\begin{array}{l}\text { AOAC Método } \\
\text { oficial } 991.14 \\
\end{array}$ \\
\hline & $\begin{array}{c}\text { Recuento de Mohos y } \\
\text { Levaduras }\end{array}$ & $\mathrm{UPC} / \mathrm{mL}$ & $<1^{*}$ & $\begin{array}{l}\text { AOAC Método } \\
\text { oficial } 997.02\end{array}$ \\
\hline & Aerobios mesofilicos. & $\mathrm{UPC} / \mathrm{mL}$ & $<1^{*}$ & $\begin{array}{l}\text { AOAC Método } \\
\text { oficial } 966.23\end{array}$ \\
\hline \multirow{3}{*}{ Muestra 2} & $\begin{array}{c}\text { Determinación de } \\
\text { Coliformes }\end{array}$ & $\mathrm{UFC} / \mathrm{mL}$ & $<1^{*}$ & $\begin{array}{l}\text { AOAC Método } \\
\text { oficial } 991.14\end{array}$ \\
\hline & $\begin{array}{c}\text { Recuento de Mohos y } \\
\text { Levaduras }\end{array}$ & $\mathrm{UPC} / \mathrm{mL}$ & $<1^{*}$ & $\begin{array}{c}\text { AOAC Método } \\
\text { oficial } 997.02\end{array}$ \\
\hline & Aerobios mesofilicos. & $\mathrm{UPC} / \mathrm{mL}$ & $<1^{*}$ & $\begin{array}{l}\text { AOAC Método } \\
\text { oficial } 966.23\end{array}$ \\
\hline \multirow{3}{*}{ Muestra 3} & $\begin{array}{c}\text { Determinación de } \\
\text { Coliformes }\end{array}$ & $\mathrm{UFC} / \mathrm{mL}$ & $<1^{*}$ & $\begin{array}{l}\text { AOAC Método } \\
\text { oficial } 991.14\end{array}$ \\
\hline & $\begin{array}{c}\text { Recuento de Mohos y } \\
\text { Levaduras }\end{array}$ & $\mathrm{UPC} / \mathrm{mL}$ & $<1^{*}$ & $\begin{array}{c}\text { AOAC Método } \\
\text { oficial } 997.02 \\
\end{array}$ \\
\hline & Aerobios mesofilicos. & $\mathrm{UPC} / \mathrm{mL}$ & $<1^{*}$ & $\begin{array}{l}\text { AOAC Método } \\
\text { oficial } 966.23\end{array}$ \\
\hline
\end{tabular}




\subsection{Evaluación sensorial del licor de cabuya}

El análisis sensorial se realizó sobre las fermentaciones y destilaciones obtenidas a partir de Furcraea cabuya var. integra. Esta variedad demostró ser la óptima para realizar las fermentaciones, utilizando un inóculo de levaduras del $1 \%$ y una suplementación de sulfato de amonio.

Luego de analizar los resultados de la prueba comparativa, el análisis de los resultados entre el mosto destilado una vez, el mosto destilado por dos ocasiones y Tequila comercial, demostró la inexistencia de diferencias significativas entre cada muestra catada. Indicando de este modo que el licor está dentro de los parámetros de aceptación de los consumidores.

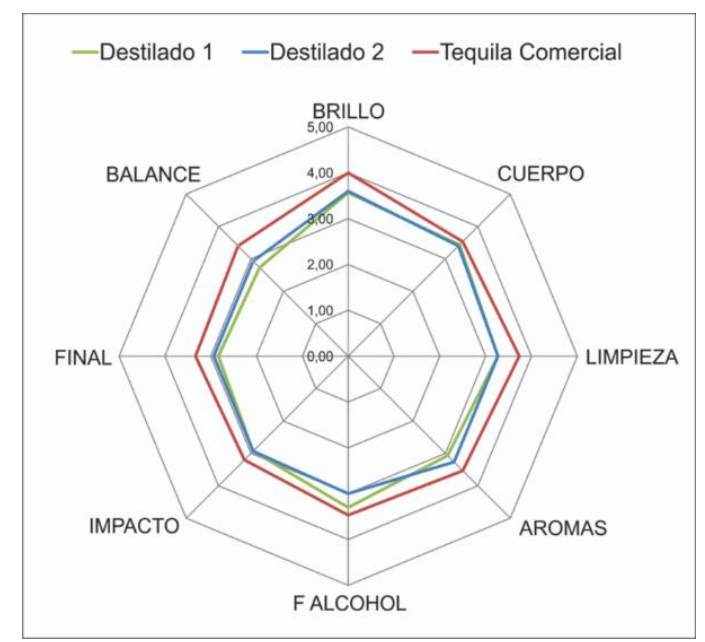

Figura 8. Evaluación sensorial del licor de F Cabuya var. integra

\section{Conclusiones}

El estado fisiológico óptimo de cosecha se asocia con la aparición del quiote o Chawarquero, previo a esto las hojas en el centro empiezan a desarrollarse más finas en comparación a las hojas antiguas. Sin embargo, al no existir estudios fenológicos de esta especie, no se pudo determinar la edad óptima de cosecha.

La variedad Furcraea cabuya var. integra posee el mayor rendimiento de mosto. El $60 \%$ de la fruta representa mosto y el $40 \%$ restante es desperdicio, mientras que la variedad Furcraea cabuya var. cabuya representa $45 \%$ de mosto y el $55 \%$ de desperdicio.

El tiempo y temperatura ideal de cocción en autoclave es de $117^{\circ} \mathrm{C}$ durante 4 horas, para garantizar la hidrólisis de la insulina en azúcares fermentables.

Furcraea cabuya var. integra es la variedad más apropiada para la obtención de una bebida alcohólica por fermentación y destilación de sus mostos.

El inóculo de levadura de Saccharomyces cerevisiae óptimo para la fermentación de la cabuya es 
la LSA comercial utilizada al $1 \% \mathrm{p} / \mathrm{v}$ con respecto al mosto.

Los mostos de cabuya no contienen los nutrientes necesarios para conseguir una fermentación total. Es necesario la adición de nitrógeno al mosto a razón de $1,5 \mathrm{~g} / \mathrm{L}$ de sulfato de amonio, cuando el inicio de la fermentación se realizó a 23,5 ํㅗix.

Se obtuvo un buen rendimiento en la producción de alcohol etílico al considerar la relación basada en la norma mexicana. Esta representa el $51 \%$ de azúcares propios de la planta y el 49\% de azúcares externos (en este caso Sacarosa). La producción de licor de cabuya rectificado y diluido a $40 \%$ vol., apto para el consumo humano, es de un $20 \%$ del volumen inicial del mosto fermentado.

El licor de cabuya se encuentra dentro de los parámetros de aceptabilidad, puede ser considerado como un tequila y está dentro de los estándares establecidos por las normas mexicanas. En Ecuador hacemos referencias a las normas INEN 375 para alcoholes rectificados, al no existir una norma para este tipo de licores.

\section{Bibliografía}

Arellano, M., Pelayo, C., Ramírez, J. \& Rodríguez, I. (2008). Characterization of kinetic parameters and the formation of volatile compounds during the tequila fermentation by wild yeast isolated from Agave juice. Journal of Industrial Microbiology Biotechnology 35, 835-841.

Meza-Freire, V. M. (2011). Obtención de una bebida isotónica nutritiva carbonatada a partir del extracto del penco de cabuya negra (Agave americana. L) (Bachelor's thesis).

Rubio-Uribe, M. E., \& Soto-Salgado, A. L. (2015). Estudio de factibilidad para la implementación de una microempresa productora de fibra de dos variedades de agave cabuya negra (Agave americana I) y agave sisal (Agave sisalana perrine) para la elaboración de artesanías en la provincia de Cotopaxi, cantón Latacunga, parroquia Belisario Quevedo, comunidad Colaguango Alto en el período 2014-2015 (Bachelor's thesis, LATACUNGA/UTC/2015).

Téllez-Mora, P., Peraza-Luna, F. A., Feria-Velasco, A., \& AndradeGonzález, I. (2012). Optimización del proceso de fermentación para la producción de tequila, 
utilizando la metodología de

superficie de respuesta

(MSR). Revista mexicana de

ingeniería química, 11(1),

163-176. 